\title{
LA TRADUCCIÓN DE LA FRASEOLOGÍA EN LOS DICCIONARIOS BILINGÜES ESPAÑOL-ITALIANO ITALIANO-ESPAÑOL
}

\author{
FEDERICA FRAGAPANE
}

\section{INTRODUCCIÓN}

El presente trabajo se enmarca dentro de la lexicografía bilingüe hispano-italiana, y más concretamente en las estrategias de traducción que se adoptan en el campo lexicográfico para establecer correspondencias entre las unidades fraseológicas (UF) de dos lenguas diferentes, como son las que nos ocupan: el español y el italiano. Hasta la fecha, para este par de lenguas se ha evidenciado cierta escasez de diccionarios especializados en fraseología, por lo que el usuario se ve obligado a recurrir casi exclusivamente a diccionarios bilingües generales. No obstante, incluso en estos diccionarios se descubren inexactitudes e incongruencias de manera usual, lo que es debido, por una parte, al tratamiento poco sistemático que se le dedica a este ámbito de la lengua y, por otra, a la verdadera dificultad de encontrar equivalencias siempre acertadas. De ahí que la inclusión de la fraseología en los diccionarios sea una tarea meticulosa que en muchas ocasiones produce resultados poco satisfactorios. De este modo, como afirma Iliná (2000: 2), «si el diccionario en cierto sentido puede ayudarnos, en las situaciones con las combinaciones fijas de palabras, él muchas veces pierde su fuerza».

En nuestro análisis se podrán distinguir dos momentos: una primera parte en la que presentaremos los diccionarios que hemos elegido como objeto de estudio, y una segunda parte en la que analizaremos las posibles estrategias de traducción de las UF.

Creemos oportuno dedicar un espacio introductorio tanto a la teoría de la fraseología como a la de la lexicografía hispano-italiana, pero, para evitar una falta de precisión derivada de la complejidad del tema, no nos detendremos en exceso en cuestiones teóricas vinculadas a la organización de la fraseología en el sistema lingüístico del español y del italiano. 


\section{DEFINICIÓN DE FRASEOLOGÍA EN ESPAÑOL E ITALIANO}

Desde una óptica contrastiva, destaca una diferencia clara entre estas dos lenguas, que se refleja en la definición de fraseología ofrecida por los diccionarios monolingües. Dos ejemplos representativos de esta disparidad son los siguientes:

fraseología (Diccionario de la Real Academia Española, 2001 —edición en CDROM, 2003-):

1. f. Conjunto de modos de expresión peculiares de una lengua, de un grupo, de una época, actividad o individuo.

2. Conjunto de expresiones intrincadas, pretenciosas o falaces.

\section{3. palabrería.}

4. Conjunto de frases hechas, locuciones figuradas, metáforas y comparaciones fijadas, modismos y refranes, existentes en una lengua, en el uso individual o en el de algún grupo.

5. Parte de la lingüística que estudia las frases, los refranes, los modismos, los proverbios y otras unidades de sintaxis total o parcialmente fijas.

fraseología (Diccionario italiano Zingarelli-Zanichelli, 2011):

1. Insieme delle frasi proprie di un determinato sistema linguistico o di una sua parte: f. italiana, f. militare / Raccolta ragionata di tali frasi.

2. Costrutto della frase proprio di una lingua o di uno scrittore.

La definición española es, sin duda, mucho más completa. En ambos casos se hace mención a un conjunto lingüístico compuesto por unidades de distintas tipologías, pero, a diferencia del español, en italiano se alude a frases en general, sin especificar de qué tipo. Nuestra conjetura de que en italiano el concepto resulta mucho más indefinido se ve confirmada por la constatación de Quiroga (2006: 41): «en italiano por fraseología se entiende todos aquellos elementos que los lexicógrafos reúnen en la microestructura de una entrada».

Esto lo ratifica Casadei (2001: 49-50), quien, a propósito de la fraseología, especifica:

L'insieme delle espressioni convenzionali e cristallizzate che costituiscono frasi fatte o modi di dire tipici di una lingua, è un insieme molto ampio, che può comprendere polirematiche e frasi idiomatiche (alla bell'e meglio, ingoiare il rospo), proverbi e detti (chi non rosica non risica, il tempo è denaro), formule (buon appetito), collocazioni (efferato delitto). Nei dizionari la fraseologia è l'insieme di frasi citate per fornire esempi dei contesti d'uso più tipici di una parola.

El hecho de que no exista una simetría perfecta entre italiano y español en cuanto a clasificación nos conduce a reconocer la dificultad de trabajar con este componente lingüístico, puesto que a los inconvenientes intralingüísticos relativos a la difícil sistematización dentro de una única lengua se suman los inconvenientes interlingüísticos que 
derivan de la imposibilidad de establecer paralelos exactos incluso entre dos lenguas tan próximas como son el español y el italiano.

En líneas generales, los diccionarios de ambas lenguas coinciden en que la fraseología se compone de frases hechas reproducidas por los hablantes de manera convencional. Se trata de unidades pluriléxicas dotadas de una gran eficacia comunicativa, puesto que son de utilidad para expresar contenidos que difícilmente se podrían transmitir mediante unidades monolexemáticas.

Carneado Moré (1985: 43) observa:

Mientras la función fundamental de las palabras consiste en expresar los conceptos, los fraseologismos, mediante una representación metafórica, caracterizan un concepto ya denominado por una palabra, lo concretizan y hacen más expresivo.

De acuerdo con Corpas Pastor (1996: 20), las UF se pueden considerar:

Unidades léxicas formadas por más de dos palabras gráficas en su límite inferior, [que] se caracterizan por una alta frecuencia de uso y de coaparición de sus elementos integrantes; por su institucionalización, entendida en términos de fijación y especialización semántica; por su idiomaticidad y variación potencial.

Por tanto, las características que definen a estas unidades son tres: su naturaleza plurilexemática, su fijación y su idiomaticidad. Para ser más precisos, la primera característica consiste en la presencia de dos o más palabras gráficas. El segundo rasgo se podría definir bien como la estabilidad de las unidades a nivel diacrónico, bien como la estabilidad de los elementos constitutivos a nivel estructural. Finalmente, la tercera característica está vinculada al plano semántico de la frase, y se refiere a que el significado fraseológico no es composicional, ya que en la mayoría de los casos no puede deducirse de la suma de los significados parciales de las distintas palabras. El significado fraseológico es en realidad total, y hacia él concurren todos los componentes léxicos que forman parte de la unidad misma. Se trata de un significado global, surgido del propio uso de la UF en un determinado contexto. Sin embargo, la idiomaticidad, a diferencia de la fijación, no es un aspecto definitorio imprescindible para que una unidad pertenezca al campo de la fraseología.

Seguidamente, y antes de adentrarnos en un apartado más específico acerca del tratamiento de las UF dentro del marco de la lexicografía, dedicaremos un breve apartado a la presentación de los diccionarios que hemos analizado.

\section{LOS DICCIONARIOS OBJETO DE NUESTRO ESTUDIO}

En 1997, el diccionario español-italiano italiano-español de la editorial Hoepli pareció eclipsar a los anteriores, que ya se habían considerado superados. Nos referimos al diccionario de Laura Tam (Tam, a partir de ahora), hito fundamental durante muchos años, y que sigue siendo una obra notable de nuestra lexicografía bilingüe. Liverani (2008) afirma que los criterios constitutivos del Tam proceden de la elección personal de la autora y de sus colaboradores. Su título original era Dizionario spagnolo-italiano. Diccionario italiano-español, pero en la edición de 2004 fue modificado por otro título con ciertas disimilitudes respecto al anterior: Grande Dizionario di Spagnolo. Gran diccio- 
nario italiano-español. Aquí manejamos el Tam de 2004, en el que al prólogo de la primera edición se añade otro prólogo adicional, que destaca la incorporación de nuevas entradas relativas a la vida diaria y al léxico hispano-americano:

En esta segunda edición los hemos registrado [los cambios] con la inclusión de nuevas entradas relativas a la vida diaria y a los sectores especializados. [...] Hemos revisado y enriquecido los americanismos para satisfacer las exigencias de quienes entran en contacto con América Latina. (2004: Prólogo).

Considerado que la finalidad principal de la obra es proporcionar equivalencias entre las dos lenguas, los destinatarios parecen ser, al menos según las declaraciones iniciales, tanto hispanohablantes como italoparlantes:

Este diccionario se dirige a un vasto público, que no es solamente el de estudiosos de italiano [y español], sino que incluye a profesionales de todos los sectores o personas que quieren satisfacer su curiosidad lingüística. (Prólogo: 2004).

En cuanto a la fraseología, podemos afirmar que se le dedica especial atención, al considerarla un componente fundamental del habla cotidiana. En el prólogo se advierte que contiene abundantes locuciones de todo tipo, coloquiales y técnicas, modernas y menos modernas. De hecho, se señala:

Se ha enriquecido el apartado de las locuciones con expresiones, sean modernas o antiguas (refranes, modismos, locuciones técnicas sectoriales, expresiones típicamente coloquiales), para ilustrar la riqueza del idioma y al mismo tiempo interpretar las exigencias del lector.

En 2005 apareció otro repertorio, Il dizionario di Spagnolo. Dizionario Spagnolo-Italiano Italiano-Spagnolo de Secundi Sañé y Giovanna Schepisi, editado por Zanichelli/Vox (que aquí designaremos con la palabraVox). Se trata de un diccionario voluminoso, que ya en el prólogo presenta una disposición en doble columna para facilitar la consulta tanto a los usuarios españoles como a los italianos. A tal finalidad responde también la inserción de unas páginas iniciales — antes de las dos secciones principales - dedicadas, por un lado, a la pronunciación y gramática españolas, y por otro a la pronunciación y gramática italianas.

En las notas preliminares se trata un asunto fundamental: se considera que nos encontramos, ahora más que nunca, en una fase en la que los productos lexicográficos están experimentando un cambio profundo. Respecto a los diccionarios de los años 50-80 del siglo $\mathrm{XX}$, han variado los formatos, las fuentes, las bases documentales, las funciones:

Se trata, en primer lugar, de un diccionario totalmente nuevo, en su estructura, en el desarrollo de los artículos, en la elección y presentación de sus contenidos. Su redacción ha coincidido en el tiempo con la difusión de Internet y ello ha permitido llegar, a través de la consulta de corpora de lengua española y de lengua italiana, de bases de datos terminológicas y de documentos de todo tipo, a la elaboración de un lemario nuevo y equilibrado, en el que tienen cabida también tecnicismos y neologismos difíciles de hallar en otros diccionarios. (Prólogo: 2005).

Con relación a la fraseología, la presentación inicial no explica gran cosa; de manera similar, la guía de uso ofrece escasas observaciones, lo que hace suponer un tratamiento superficial del universo fraseológico. 
Presentemos finalmente el diccionario de Garzanti (GA, a partir de ahora). En 2007, esta editorial italiana contaba con diccionarios de gran tamaño, como los bilingües de inglés, de alemán y de francés. En el caso del español, existían solo el formato pequeño y el formato mediano, nacidos respectivamente en 2004 y 2007. Para que saliera a la luz el formato grande, núcleo de nuestra atención en este trabajo, hubo que esperar a 2009. En la cubierta figura el título: Garzanti Spagnolo; y unas páginas después: Spagnolo-Italiano Italiano-Spagnolo. Basándonos en este título, y aunque no se especifique nada en cuanto a los destinatarios, inferimos en seguida que la obra aspira a ser unidireccional, y que, además, se dirige de manera especial a estudiantes italoparlantes. Este hecho se deduce de que al final del repertorio se incluyan varias tablas de conjugación verbal que contienen verbos españoles. Otros indicios se desprenden de la presentación inicial que, a pesar de estar escrita en ambas lenguas, manifiesta una clara orientación unidireccional:

El Gran Diccionario Garzanti de Español está concebido a partir de la lengua hablada y escrita en España, además de contener una relación extensa de los términos y las locuciones utilizados en el ámbito hispanoamericano. [...] Algunas notas culturales en la sección español-italiano proporcionan interesantes informaciones históricas y sobre las costumbres españolas. (2009: Prólogo).

Acerca de la fraseología no se proporciona gran información en el apartado inicial; aun así, en la cubierta se garantiza la inclusión de un elemento imprescindible, a saber, las «espressioni della lingua parlata, i modi di dire e le forme gergali».

En suma, según la información aportada en las notas preliminares de estos diccionarios, se constata la ausencia de criterios aclaratorios y específicos que puedan ser de utilidad para el usuario en la consulta de las UF. Es evidente que la guía es muy aproximada. Por ejemplo, en el prólogo del Tam se alude solamente al orden alfabético con el que las unidades han sido introducidas: «Al exponerlas hemos decidido seguir el orden alfabético, porque criterios diferentes hubieran conllevado una consulta más complicada». El GA y el Vox, en este aspecto, resultan aún más superficiales. Concretamente, en referencia a la fraseología, hallamos lo siguiente:

- GA: Locuciones, modismos y refranes aparecen destacados en negrita e insertados al final del artículo, en un apartado introducido por el signo $\bullet$.

- Vox: Compuestos y colocaciones; locuciones verbales; locuciones prepositivas; locuciones adverbiales; otros modismos (con sus correspondientes traducciones). Esta sección sucede al tratamiento léxico de cada subentrada, y precede al apartado dedicado a las variantes morfológicas del lema. Cada grupo de locuciones aparece marcado mediante el signo $(\bullet)$. En esta sección se ha incluido también un número de proverbios, introducidos por la abreviación PROV., representando estos proverbios usos particulares de los lemas. Se ha tratado de precisar para cada uno de ellos el proverbio correspondiente en la otra lengua.

Se puede concluir que, por regla general, en los prólogos se alude solo a la ubicación de la fraseología en el plano de la microestructura, pero no se especifica ningún criterio ni de clasificación ni de lematización. 


\section{PROBLEMAS DE TRADUCCIÓN}

En la dinámica que se pone en funcionamiento cuando se emprende el aprendizaje de una lengua extranjera, las unidades fraseológicas representan un capítulo fundamental, dado que constituyen un aspecto imprescindible de la comunicación. Como subraya Olza Moreno (2006: 2), «en esta parcela de las lenguas se depositan muy frecuentemente las ideas, opiniones y creencias que sobre la realidad desarrolla cada comunidad de habla». De ahí que no resulte fácil englobarlas en los diccionarios bilingües y trasladarlas con éxito de una lengua a otra. Al fin y al cabo, su traducción equivale a una transmisión de cultura, con todo lo que esto conlleva.

Tras la identificación de una UF, el primer paso para su comprensión, si el contexto no resulta suficiente para interpretarla, consiste en indagar posibles correspondencias en el sistema léxico de la lengua meta. Por consiguiente, la transposición a otra lengua se convierte en la estrategia más importante para aproximarse a este material lingüístico en una L2.

Desde el pasado siglo, la traducción de la fraseología se ha convertido en un apasionante objeto de estudio por parte de muchos investigadores, revelándose como una materia que exige un tratamiento particular. Más concretamente, se trata de un proceso de interpretación que varía en función de diversos factores y que puede incluso cambiar dependiendo del autor. Torop (1995: 83) enfatiza que «in qualsiasi processo di traduzione inevitabilmente tocca parlare di interrelazioni tra elementi traducibili, tralasciabili, modificabili e aggiungibili».

En principio, el margen de variabilidad interpretativa en la traducción de la fraseología tiene un determinado límite, por hallarse vinculado, en muchas ocasiones, a equivalencias fijas. Sin embargo, en cualquier caso, es el autor de la traducción quien decide qué elementos traducir y cómo traducirlos, pues, como observa García Yebra (1983: 101), «el traductor ha de saber elegir, entre varias soluciones, la más adecuada».

Para apreciar lo complejo que es seleccionar las equivalencias fraseológicas de otra lengua, se pueden considerar, a modo de ejemplo, los refranes. Estas unidades representan una categoría fraseológica bastante problemática y están traducidos del italiano al español de manera muy diferente según de qué diccionario consultado se trate. Para el refrán italiano Donne e buoi dei paesi tuoi, se pueden generar muy diversas traducciones, debido a la falta de una correspondencia total con la lengua española. El Vox ofrece como equivalente Buey y tu mujer, de tu tierra deben ser, mientras que el Tam propone Si quieres bien casar, casa con tu igual; el GA, por su parte, propone: Mujer y tocino, tómalos del vecino. Es muy ilustrativo también el ejemplo Tra moglie e marito non mettere il dito, que se traduce con dos unidades completamente distintas: en el GA se encuentra Entre marido y mujer nadie se debe meter, mientras que en el Tam aparece No entremeterse en familia ajena, que, entre otros aspectos, de refrán pasa a transformarse en locución.

\subsection{Estrategias de traducción}

La noción de equivalencia es un concepto clave en traductología. Quiroga (2006: 140-141) establece una distinción entre equivalencia y correspondencia. Se entiende por correspondencia la simetría que se puede establecer en el plano del sistema, esto es, 
cuando dos unidades coinciden desde un punto de vista estrictamente lingüístico. Con la noción de equivalencia, en cambio, se hace referencia a la dimensión pragmática y a un contexto situacional determinado. Se puede colegir, por tanto, que las correspondencias no siempre coinciden con las equivalencias. En nuestro estudio, sin embargo, optaremos por utilizar los dos términos indistintamente, tratándolos como sinónimos y asumiendo para ambos el significado de 'equivalencia', es decir, la traducción de una situación mediante otra perfectamente adecuada desde todos los puntos de vista. Obviamente, en los diccionarios bilingües se deberían integrar ambos conceptos, ya que el objetivo primordial debe ser establecer una equivalencia completa.

Navarro (2008: 141-142) precisa:

El lexicógrafo, en principio, puede contar con dos tipos de equivalencia que dependen de su campo de aplicación: la equivalencia semántica o funcional, finalidad de la fraseología contrastiva, que podemos definir como la equivalencia del significado de las unidades lingüísticas [...] y la equivalencia comunicativa, situacional o textual, meta de la traductología, en la que juegan un papel importante aspectos pragmáticos como la situación comunicativa, registro, marcas estilísticas, etc.

La técnica más usual de traducción de unidades fraseológicas puede generar tres tipos de equivalencias: total, parcial o nula. La equivalencia es total cuando la unidad fraseológica de la lengua de origen corresponde a otra, en la lengua meta, que presenta el mismo significado, la misma estructura, la misma carga pragmática. Es parcial cuando los equivalentes difieren en algún aspecto, relacionado bien con la base metafórica, bien con la estructura formal. Por último, es nula cuando no se logra determinar ninguna correspondencia en la L2. En todas estas situaciones pueden producirse casos de infratraducción o sobretraducción. La primera se origina cuando el texto meta carece de ciertos aspectos estilísticos o semánticos presentes en el texto de origen, mientras que la sobretraducción surge cuando en el texto meta aparecen aspectos no recogidos en el texto original.

Existe también otro tipo de equivalencia, denominada aparente, por agrupar unidades fraseológicas que se consideran idénticas por la forma, pero que, en realidad, son semánticamente diferentes.

En líneas generales, el traductor puede recurrir a varias estrategias para traducir una unidad de una lengua a otra. Quiroga (2006: 145) distingue los siguientes procedimientos:

1. Traducción mediante otra UF que presenta la misma forma y el mismo significado que la del TO (texto origen).

2. Traducción mediante una UF equivalente en el significado, pero diferente en algún otro aspecto.

3. Traducción mediante una paráfrasis.

4. Traducción de una UF por una unidad léxica simple equivalente en significado.

5. Traducción por omisión.

6. Traducción por compensación.

7. Préstamo.

8. Calco.

9. Nota del traductor. 
En los diccionarios bilingües, las prácticas más habituales son las cuatro primeras mencionadas, puesto que resulta indispensable facilitar equivalentes que traten de expresar el mismo significado en la lengua de destino. No es conveniente ni omitirlos ni sustituirlos por una nota del traductor. Incluso cuando no se mantiene el carácter fraseológico, es necesario asegurar un equivalente que se pueda aplicar en los mismos contextos comunicativos que los de la lengua de origen. Por esta razón, es aconsejable evitar tanto el calco, que consiste en la creación de un neologismo en la lengua meta mediante una traducción literal, como el préstamo, que se origina al procurar conservar la unidad de la lengua meta, escribiéndola simplemente en cursiva.

Ahora bien, para que nuestro trabajo no se limite a un plano exclusivamente teórico, procedemos a ejemplificar las estrategias traductológicas que figuran con mayor frecuencia en los diccionarios de nuestra bibliografía.

\subsection{Ejemplificaciones de estrategias traductológicas extraídas de nuestros diccionarios}

Existen equivalencias que preservan tanto la forma como el significado de la unidad de origen:

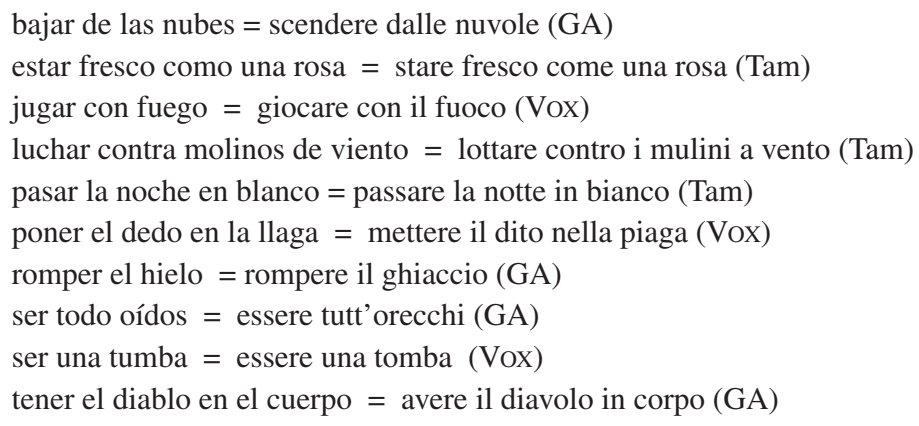

Sin embargo, es necesario señalar que una equivalencia total en la L2 no es garantía de una traducción adecuada, ya que la expresión resultante podría ser menos familiar que otra, aunque esta última, más usual, resulte menos literal. Es decir, no es necesario reproducir una traducción literal en toda ocasión cuando se puede recurrir a expresiones más acertadas, pese a que no se trate de equivalencias idénticas. A modo de ejemplo:

(Esp.) En boca cerrada no entran moscas = (It.) In bocca chiusa non entrano mosche (Vox)

Esta traducción, realizada palabra por palabra, no resulta convincente al no representar un refrán de uso común en italiano. Habría expresiones más adecuadas, como $\grave{E}$ meglio tacere, Acqua in bocca, Dalla mia bocca non uscirà parola. Siempre en el Vox, lavorare come un negro, traducción literal de trabajar como un negro, es una expresión italiana de uso menos frecuente que lavorare come un mulo/bue. Asimismo, la locución stringere $i$ cordoni della borsa, propuesta en correspondencia de darlechar otro nudo a la bolsa, se revela como otra estructura poco utilizada respecto a tagliare le spese, que, aunque muy alejada de la forma original, logra a la perfección la equivalencia comunicativa. 
Otro ejemplo puede ser Lo que no mata, engorda, traducida con Quello che non strozza ingrassa, en este caso una equivalencia derivada de una traducción literal que produce un significado poco comprensible en italiano.

En cambio, en otras ocasiones resulta anecdótico el hecho de que, aun pudiéndose proponer una equivalencia total, el autor no se inclina por ella:

It. andarsene con la coda tra le gambe $=$ Esp. llevar recado $($ Tam $)$

Aquí se podría haber recurrido a la equivalencia total irse con el rabo entre las piernas.

2) El traductor puede también establecer equivalencias en el nivel del sistema, transmitiendo un significado idéntico mediante unidades que mantienen el carácter fraseológico, pero difieren en algún aspecto léxico o sintáctico.

dar una en el clavo y ciento en la herradura = azzaccarne una su cento $($ Tam $)$

lo que no mata engorda $=$ tutto fa brodo $($ Tam $)$

perder la hebra $=$ perdere il filo del discorso $($ Tam $)$

parecerse como un huevo a una castaña $=$ somigliarsi come il giorno e la notte $(\mathrm{GA})$

Otras veces, en cambio, el carácter fraseológico se mantiene, pero varía la clasificación de la unidad. Por ejemplo, una locución verbal española llega a ser una colocación en italiano:

Esp. trabajar como un enano = It. lavorare sodo, da matti $(\mathrm{GA})$

Por lo general, la falta de precisión que se observa en los diccionarios casi siempre está relacionada con el tratamiento del significado, terminándose así por proporcionar en muchas ocasiones equivalentes que por el significado que vehiculan no evidencian un uso real en la L2:

agarrarse a un clavo ardiendo $=$ attaccarsi ai rasoi (Tam)

dormir a pierna suelta $=$ dormire della grossa $($ Tam $)$

no pegar ni con cola $=$ entrarci come il cavolo a merenda $(\mathrm{GA})$

tener las espaldas muy anchas $=$ avere le spalle quadrate/grosse $(\mathrm{Tam})$

Las expresiones elegidas aquí como equivalencias no son muy corrientes en italiano, y algunas incluso, como dormire della grossa, están desprovistas de eficacia comunicativa por ser poco actualizadas. Es más, para el mismo contexto se podría proporcionar alguna expresión más usual, como dormire come un ghiro; o avere le spalle larghe antes que avere le spalle quadrate. Asimismo, la locución agarrarse a un clavo ardiendo se traduce mediante un equivalente poco apropiado, tanto en el Tam como en el GA:

Esp. agarrarse a un clavo ardiendo $=$ It. essere disposto a fare qualsiasi cosa.

En realidad, la traducción más adecuada sería arrampicarsi sugli specchi.

Son frecuentes los errores de interpretación, que conducen a traducciones incorrectas, en absoluto relacionadas con el significado expresado por el equivalente de la L2. Es el caso de poner buena / mala cara, que el GA traduce como fare buona / cattiva acco- 
glienza, una equivalencia que no solo no corresponde al significado de la frase española, sino que además carece de sentido en italiano.

El Tam, en particular, comete numerosas inexactitudes en cuanto al significado, al proporcionar equivalentes poco convincentes desde una óptica italoparlante, como puede observarse en los siguientes casos:

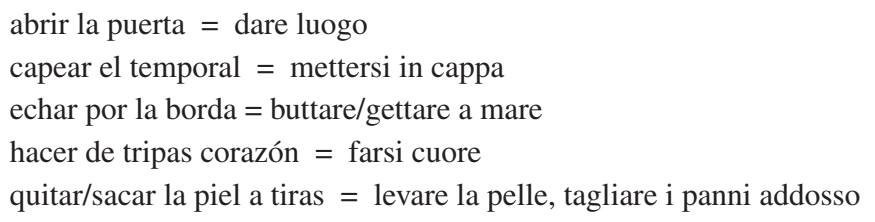

3) En otras ocasiones, en la traducción se transfiere el significado de la UF mediante una sola unidad léxica. Concretamente, se trata de unidades fraseológicas españolas que se hacen corresponder con unidades monolexemáticas en italiano. Obviamente, en dicho proceso resulta más problemática la descodificación de un idioma a otro. Algunos ejemplos de este fenómeno son:

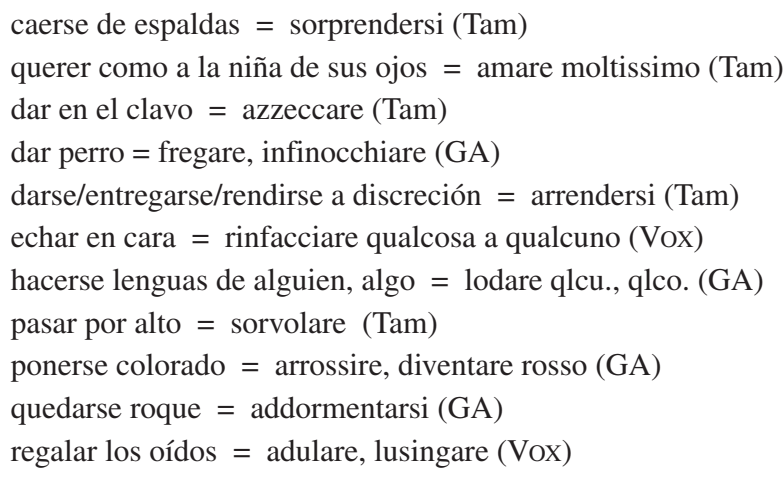

La técnica aplicada a estos ejemplos constituye una estrategia de traducción muy frecuente en nuestros diccionarios, que demuestra, una vez más, la enorme distancia entre la lengua española y la lengua italiana respecto al uso de la fraseología. Además, el hecho de que en italiano los términos equivalentes se materialicen frecuentemente como simples verbos implica que no se recurre a la fraseología tan a menudo como en español.

De igual manera, cuando no existe una correspondencia exacta se puede recurrir a una reformulación parafrástica. Así pues, una vez más, la dificultad en el tratamiento del significado en la L2 produce la pérdida del carácter fraseológico de la unidad de la L1:

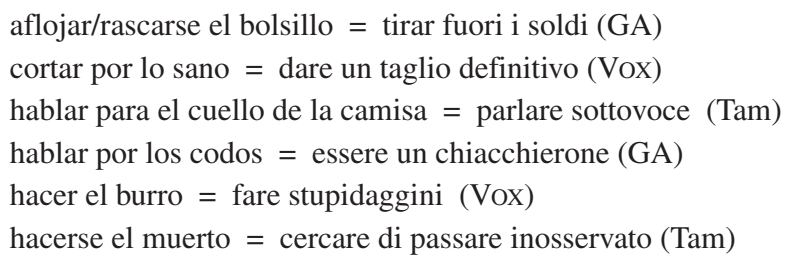


mirarse a la sombra $=$ essere presuntuoso $($ Tam $)$

no dar pie con bola $=$ non azzeccarne una $($ Vox $)$

oler a cuerno quemado = fare una brutta impressione, insospettire (Vox)

poner un granito de arena $=$ dare il proprio contributo $(\mathrm{Tam})$

remachar el clavo $=$ peggiorare le cose $(\mathrm{GA})$

Realmente no se puede comprender el motivo para ofrecer paráfrasis incluso cuando se podría contar con UF equivalentes:

Esp. hincar los codos = It. studiare di più (GA)

Aquí se podría haber empleado sgobbare sui libri, expresión fraseológica más habitual, con un significado afín y más eficaz desde un punto de vista comunicativo.

Esp. cortar por lo sano = It. tagliare di netto $($ Tam $)$

En este caso podría haberse propuesto darci un taglio, equivalente muy apropiado tanto por su carga expresiva como por su frecuencia de uso.

Bien es verdad que dentro de un mismo diccionario se puede adoptar más de una estrategia de traducción para la misma unidad, y, por consiguiente, puede aparecer más de una equivalencia en la L2. Ejemplos son el Tam y el Vox, que, según cuál sea el lema buscado, cortar o sano, ofrecen traducciones distintas:

\begin{tabular}{|c|c|c|}
\hline Tam & GA & Vox \\
\hline $\begin{array}{l}\text { - Cortar por lo sano: tagliare } \\
\text { di netto. } \\
\text { - Cortar por lo sano: darci un } \\
\text { taglio. }\end{array}$ & $\begin{array}{l}\text { - Cortar por lo sano: dare un } \\
\text { taglio netto, darci un taglio. }\end{array}$ & $\begin{array}{l}\text { - Cortar por lo sano: dare un } \\
\text { taglio definitivo. } \\
\text { - Cortar por lo sano: tagliare } \\
\text { la testa al toro. }\end{array}$ \\
\hline
\end{tabular}

Cuando no existe simetría total y se proporciona más de un equivalente, puede suceder que el conjunto abarque bien unidades fraseológicas, bien unidades léxicas simples. A continuación se muestran varios ejemplos de esta opción:

Esp. andarse por las ramas = uscire dal seminato, divagare $($ Vox $)$ comerse los niños crudos $=$ essere un orco, incutere paura $($ TAM) dar en el clavo $=$ azzeccare, colpire nel segno, centrare in pieno (GA)

Por otra parte, la traducción fraseológica puede ser el resultado de distintas estrategias según cuál sea el diccionario. Así, podemos encontrar:

dedicare troppo tempo a qlco. (GA)

faro una sviolinata a qualcuno (Tam)

adulare (Vox)

scendere nell'arena/in campo (Tam)

$=\quad$ dar coba

scendere nell' arena/scendere in capo (GA)

gettarsi nella mischia (Vox)

$$
=\quad \begin{aligned}
& \text { echarse al ruedo (Tam) } \\
& \text { salir (o saltar) al ruedo (GA) } \\
& \text { echarse al ruedo (Vox) }
\end{aligned}
$$


Respecto al último ejemplo, debe advertirse que el Vox, s.v. ruedo, registra también la expresión fraseológica salir o saltar al ruedo. Por consiguiente, es evidente que, dentro de un mismo diccionario, no se puede suponer un tratamiento sistemático de las equivalencias propuestas.

En conformidad con el planteamiento contrastivo de nuestro estudio, procedemos a comprobar a qué tipo de traducción dan prioridad nuestros diccionarios, para averiguar el tipo de ayuda con la que puede contar el usuario no nativo. Dentro de este marco, lo que más nos interesa es el análisis de dos aspectos: en primer lugar, qué estrategias se suelen utilizar para traducir unidades que no tienen equivalencias completas en italiano y, en segundo lugar, qué diferencias se observan entre los procedimientos aplicados a los diccionarios de nuestro repertorio.

Así pues, compararemos el tratamiento lexicográfico de algunas UF que tienen como rasgo común la lexicalización de un componente referido a una parte del cuerpo.

\begin{tabular}{|c|c|c|c|}
\hline LOC & Tam & Vox & GA \\
\hline ir de boca en boca & $\begin{array}{l}\text { essere sulla bocca di } \\
\text { tutti }\end{array}$ & $\begin{array}{l}\text { passare di bocca in boc- } \\
\text { ca, essere sulla bocca di } \\
\text { tutti }\end{array}$ & $\begin{array}{l}\text { andare (o passare) di } \\
\text { bocca in bocca }\end{array}$ \\
\hline andar con cien ojos & $\begin{array}{l}\text { andare coi piedi di } \\
\text { piombo }\end{array}$ & - & $\begin{array}{c}\text { stare allerta, avere cento } \\
\text { occhi }\end{array}$ \\
\hline $\begin{array}{c}\text { aplastar/planchar la } \\
\text { oreja }\end{array}$ & dormire & dormire & dormire, andare a nanna \\
\hline caer de pie & cadere in piedi & $\begin{array}{l}\text { cascare in piedi, cadere } \\
\text { in piedi }\end{array}$ & - \\
\hline caer de espaldas & sorprendersi & - & $\begin{array}{l}\text { rimanere di stucco, } \\
\text { rimanere di sale, cadere } \\
\text { dalle nuvole }\end{array}$ \\
\hline $\begin{array}{c}\text { caérsele el pelo a al- } \\
\text { guien }\end{array}$ & ssere in castigo, sentirle & - & $\begin{array}{l}\text { ricevere quel che si me- } \\
\text { rita, ricevere una puni- } \\
\text { zione }\end{array}$ \\
\hline calentar la cabeza & arrovellarsi il cervello & $\begin{array}{l}\text { rompersi la testa, scer- } \\
\text { vellarsi }\end{array}$ & $\begin{array}{l}\text { arrovellarsi, preoccupar- } \\
\text { si }\end{array}$ \\
\hline comerse los codos & mangiarsi le mani & $\begin{array}{l}\text { [de hambre:] fare la } \\
\text { fame }\end{array}$ & $\begin{array}{l}\text { [de hambre:] fare la } \\
\text { fame }\end{array}$ \\
\hline costar un ojo de la cara & $\begin{array}{c}\text { costare un occhio della } \\
\text { testa }\end{array}$ & $\begin{array}{c}\text { costare un occhio della } \\
\text { testa }\end{array}$ & - \\
\hline cojear del mismo pie & $\begin{array}{c}\text { essere dello stesso } \\
\text { stampo, } \\
\text { Dio li fa e poi li accop- } \\
\text { pia }\end{array}$ & - & avere gli stessi difetti \\
\hline
\end{tabular}




\begin{tabular}{|c|c|c|c|}
\hline LOC & Tam & Vox & GA \\
\hline $\begin{array}{c}\text { cruzarse de manos/bra- } \\
\text { zos }\end{array}$ & stare a vedere & - & $\begin{array}{c}\text { incrociare le braccia, } \\
\text { stare con le mani in } \\
\text { mano }\end{array}$ \\
\hline $\begin{array}{c}\text { coger/pillar con las ma- } \\
\text { nos en la masa }\end{array}$ & $\begin{array}{c}\text { cogliere con le mani nel } \\
\text { sacco }\end{array}$ & $\begin{array}{l}\text { cogliere con le mani nel } \\
\text { sacco }\end{array}$ & $\begin{array}{c}\text { [func. adv.] } \\
\text { con le mani nel sacco }\end{array}$ \\
\hline dar pie & dare adito & $\begin{array}{c}\text { dare adito, } \\
\text { dare argomento, dare } \\
\text { motivo, } \\
\text { prestare il fianco }\end{array}$ & $\begin{array}{l}\text { dare adito, } \\
\text { dare retta }\end{array}$ \\
\hline dormir a pierna suelta & dormire saporitamente & $\begin{array}{l}\text { dormire saporitamente, } \\
\text { dormire come un ghiro }\end{array}$ & $\begin{array}{l}\text { dormire della grossa, } \\
\text { dormire come un ghiro }\end{array}$ \\
\hline hacer oídos sordos & $\begin{array}{l}\text { fare orecchie da mer- } \\
\text { cante }\end{array}$ & $\begin{array}{l}\text { fare orecchie da mer- } \\
\text { cante }\end{array}$ & $\begin{array}{l}\text { fare orecchie da mer- } \\
\text { cante }\end{array}$ \\
\hline ir de cabeza & $\begin{array}{c}\text { [por algo] } \\
\text { avere una voglia matta } \\
\text { di qualcosa }\end{array}$ & essere indaffarato & - \\
\hline $\begin{array}{c}\text { meter las narices en } \\
\text { algo }\end{array}$ & ficcare/cacciare il naso & ficcare il naso & ficcare il naso in qlco. \\
\hline nacer de pie & nascere con la camicia & $\begin{array}{l}\text { [haber nacido de pie: } \\
\text { essere nato con la } \\
\text { camicia] }\end{array}$ & nascere con la camicia \\
\hline no dar el brazo a torcer & $\begin{array}{l}\text { non spostarsi di un pas- } \\
\text { so, una virgola }\end{array}$ & non cedere, ostinarsi & non darla vinta \\
\hline $\begin{array}{c}\text { no decir esta boca es } \\
\text { mía }\end{array}$ & $\begin{array}{l}\text { non aprire bocca, non } \\
\text { dire bè }\end{array}$ & $\begin{array}{c}\text { non aprire bocca, } \\
\text { stare zitto }\end{array}$ & non aprire bocca \\
\hline no mover un dedo & non muovere un dito & non alzare un dito & $\begin{array}{l}\text { non alzare (o non muo- } \\
\text { vere) un dito per qlcu., } \\
\text { qlco. }\end{array}$ \\
\hline $\begin{array}{c}\text { no tener un pelo de ton- } \\
\text { to }\end{array}$ & essere molto sveglio & $\begin{array}{l}\text { non essere affatto sce- } \\
\text { mo, non essere scemo }\end{array}$ & $\begin{array}{c}\text { per niente } \\
\text { essere sveglio }\end{array}$ \\
\hline poner cara de perro & guardare in cagnesco & $\begin{array}{c}\text { [a cara de perro: con } \\
\text { durezza, a brutto muso] }\end{array}$ & - \\
\hline $\begin{array}{c}\text { poner los pies en polvo- } \\
\text { rosa }\end{array}$ & darsela a gambe & darsela a gambe & darsela a gambe \\
\hline romperse la cara por & difendere a spada tratta & - & - \\
\hline
\end{tabular}




\begin{tabular}{|c|c|c|c|}
\hline LOC & Tam & Vox & GA \\
\hline sellar los labios & sigillare le labbra & chiudere la bocca a qlc. & $\begin{array}{c}\text { sigillare le labbra, man- } \\
\text { tenere un segreto }\end{array}$ \\
\hline tener bigotes & essere un duro & - & non essere da poco \\
\hline tener brazo & $\begin{array}{c}\text { [tener buenos brazos, } \\
\text { avere buone braccia] }\end{array}$ & - & - \\
\hline $\begin{array}{c}\text { tener más cara que es- } \\
\text { palda }\end{array}$ & $\begin{array}{c}\text { non conoscere la ver- } \\
\text { gogna }\end{array}$ & - & $\begin{array}{c}\text { avere una bella faccia } \\
\text { tosta }\end{array}$ \\
\hline $\begin{array}{c}\text { vivir uno por sus manos } \\
\text { vivere del proprio lavo- } \\
\text { ro/sulle proprie braccia }\end{array}$ & $\begin{array}{c}\text { vivere sulle proprie } \\
\text { braccia }\end{array}$ & - \\
\hline
\end{tabular}

Concretamente, las estrategias utilizadas son las siguientes:

\begin{tabular}{|c|c|c|c|c|}
\hline LOC & $\begin{array}{c}\text { Mismo } \\
\text { significado, } \\
\text { misma forma }\end{array}$ & $\begin{array}{c}\text { Mismo } \\
\text { significado, } \\
\text { forma distinta }\end{array}$ & $\begin{array}{c}\text { Traducción } \\
\text { mediante unidad } \\
\text { léxica simple }\end{array}$ & $\begin{array}{c}\text { Traducción } \\
\text { mediante } \\
\text { perífrasis }\end{array}$ \\
\hline ir de boca en boca & GA, Vox & Tam, Vox & & \\
\hline andar con cien ojos & GA & Tam, GA & & \\
\hline $\begin{array}{c}\text { aplastar/planchar } \\
\text { la oreja }\end{array}$ & & & Tam, Vox, GA & GA \\
\hline caer de pie & Tam, Vox & & & \\
\hline caer de espaldas & & GA & Tam & \\
\hline $\begin{array}{c}\text { caérsele el pelo a } \\
\text { alguien }\end{array}$ & & & Tam & Tam, GA \\
\hline $\begin{array}{c}\text { calentarse la } \\
\text { cabeza }\end{array}$ & & $\begin{array}{l}\text { Vox, Tam, Vox, } \\
\text { GA }\end{array}$ & & \\
\hline comerse los codos & & Tam, Vox, GA & & \\
\hline $\begin{array}{c}\text { costar un ojo de la } \\
\text { cara }\end{array}$ & Tam, Vox & & & \\
\hline $\begin{array}{c}\text { cojear del mismo } \\
\text { pie }\end{array}$ & & Tam & GA & \\
\hline $\begin{array}{c}\text { cruzarse de } \\
\text { manos/brazos }\end{array}$ & & GA & Tam & \\
\hline
\end{tabular}




\begin{tabular}{|c|c|c|c|c|}
\hline LOC & $\begin{array}{c}\text { Mismo } \\
\text { significado, } \\
\text { misma forma }\end{array}$ & $\begin{array}{c}\text { Mismo } \\
\text { significado, } \\
\text { forma distinta }\end{array}$ & $\begin{array}{c}\text { Traducción } \\
\text { mediante unidad } \\
\text { léxica simple }\end{array}$ & $\begin{array}{l}\text { Traducción } \\
\text { mediante } \\
\text { perífrasis }\end{array}$ \\
\hline $\begin{array}{l}\text { coger/pillar con las } \\
\text { manos en la masa }\end{array}$ & & Tam, Vox, GA & & \\
\hline dar pie & & Tam, Vox, GA & & Vox \\
\hline $\begin{array}{c}\text { dormir a pierna } \\
\text { suelta }\end{array}$ & & Vox, GA & & Tam, Vox \\
\hline hacer oídos sordos & & Tam, Vox, GA & & \\
\hline ir de cabeza & & & & Vox, Tam \\
\hline $\begin{array}{c}\text { meter las narices } \\
\text { en algo }\end{array}$ & & Tam, Vox, GA & & \\
\hline nacer de pie & & Tam, Vox, GA & & \\
\hline $\begin{array}{c}\text { no dar el brazo a } \\
\text { torcer }\end{array}$ & & Tam, GA & Vox & \\
\hline $\begin{array}{c}\text { no decir esta boca } \\
\text { es mía }\end{array}$ & & Tam, Vox, GA & Vox & \\
\hline no mover un dedo & & Tam, Vox, GA & & \\
\hline $\begin{array}{l}\text { no tener un pelo de } \\
\text { tonto }\end{array}$ & & & & Tam, Vox, GA \\
\hline $\begin{array}{c}\text { poner cara de } \\
\text { perro }\end{array}$ & & Tam, Vox & & Vox \\
\hline $\begin{array}{c}\text { poner los pies en } \\
\text { polvorosa }\end{array}$ & & Tam, Vox, GA & & \\
\hline $\begin{array}{c}\text { romperse la cara } \\
\text { por }\end{array}$ & & Tam & & \\
\hline sellar los labios & Tam, GA & Vox & & GA \\
\hline tener bigotes & & GA & & Tam \\
\hline tener brazo & & Tam & & \\
\hline $\begin{array}{c}\text { tener más cara que } \\
\text { espalda }\end{array}$ & & GA & & Tam \\
\hline $\begin{array}{c}\text { vivir uno por sus } \\
\text { brazos }\end{array}$ & & Tam, Vox & & Tam \\
\hline
\end{tabular}


Se nota que, aun cuando los diccionarios coincidan en utilizar la misma estrategia de traducción, si no es la de la equivalencia total, puede haber en todo caso diferencias entre un diccionario y otro. Por ejemplo, la unidad no tener un pelo de tonto se traduce con la misma estrategia tanto en el Tam como en los otros, solo que el resultado cambia, ya que aun tratándose de producir el mismo significado mediante paráfrasis, se eligen dos maneras diferentes: en el Tam y en el GA se elige la traducción essere molto sveglio, que equivale a decir ser muy listo; mientras que en el Vox la misma estrategia da lugar a otro rodeo de palabras, non essere affatto scemo, que significa no ser tonto en absoluto. Pero hay que tener cuidado, y los cotejos de este tipo ayudan a destacar eventuales incongruencias. Si tomamos el caso de cojear del mismo pie, nos percatamos de que también en este caso se podría hablar de la misma estrategia adoptada en distintos diccionarios, es decir, mismo significado, forma distinta, pero lo sorprendente es que las equivalencias posibles essere dello stesso stampo y Dio li fa e poi li accoppia comunican matices semánticos distintos que no pueden considerarse sinonímicos desde una perspectiva italoparlante.

A partir de estos datos se desprende que establecer equivalencias semánticas con otra lengua puede convertirse en tarea ardua. En especial, las dificultades resultan de los casos que no tienen correspondencias unívocas entre las dos lenguas en cuestión. Así, en el caso de unidades como costar un ojo de la cara, no mover un dedo, poner los pies en polvorosa, los distintos diccionarios concuerdan por tratarse de equivalencias totales o estables, mientras que para ejemplos como calentarse la cabeza, tener bigotes, tener más cara que espalda, es más fácil que difieran al poder generarse varias equivalencias más o menos apropiadas según el contexto.

Por lo general, se alternan casos en los que se adopta una perspectiva que da preferencia al aspecto formal de la unidad en cuestión con otros casos en los que se toma en especial consideración la dimensión pragmática de la unidad, renunciando así a posibles equivalencias totales. En resumen, es inevitable que distintos diccionarios acaben ofreciendo equivalencias distintas. Pero debería evitarse que dentro de un mismo diccionario se caiga en la trampa de incongruencias semánticas o léxicas que puedan desorientar al usuario.

\section{LAS EQUiVALENCIAS APARENTES}

Las equivalencias aparentes se pueden definir como falsos amigos en el ámbito de la fraseología. Se trata de unidades que, para dos lenguas diferentes, presentan la misma estructura y el mismo significado denotativo, pero dos significados figurados distintos. Por consiguiente, a primera vista son idénticas, aunque en realidad no muestran correspondencias exactas desde un punto de vista semántico. Se convierten en un motivo de confusión, por inducir al lector a considerarlas equivalencias totales en la L2.

El tratamiento de estas unidades en los diccionarios bilingües suele ser contradictorio, porque en algunas ocasiones no se toma en cuenta la discrepancia real entre forma y significado y se acaba por proporcionar equivalentes semánticos inapropiados, que producen correspondencias erróneas. Efectivamente, la habilidad del traductor estriba en no confundir la forma y el significado de estas expresiones, así como en no tratar de igua- 
larlas en todo caso para ambas lenguas. En lugar de ello, se deben crear las condiciones adecuadas para que el usuario las interprete de manera correcta. A continuación proponemos un pequeño glosario de equivalencias aparentes, con la finalidad de analizar su tratamiento en los diccionarios que les siguen:

1. Esp. a brazo = de forma manual.

It. a braccio $=$ de manera improvisada.

2. Esp. abrir boca $=$ despertar el apetito con algún alimento.

It. aprire bocca $=$ hablar.

3. Esp. a manos llenas $=$ generosamente.

It. a mani piene/vuote = con las manos llenas o vacías de lo que se deseaba.

4. Esp. en las barbas [de alguien] = en su presencia.

It. alla barba [di qualcuno] $=$ a pesar de alguien.

5. Esp. mano a mano = entre dos personas solamente, en colaboración, en competición.

It. a mano a mano = gradualmente, a medida que.

6. Esp. meter mano = sobar, tocar con intención sexual.

It. mettere mano = intervenir en algo.

7. Esp. ir cabeza abajo = decaer, arruinarse.

It. andare a testa bassa $=$ humillarse por tener vergüenza de uno mismo.

\begin{tabular}{|c|c|c|c|c|c|}
\hline \multicolumn{2}{|c|}{ TAM } & \multicolumn{2}{|c|}{ GA } & \multicolumn{2}{|c|}{ VOX } \\
\hline ESP-IT & IT-ES & PESP-IT & IT-ESP & ESP-IT & IT-ESP \\
\hline $\begin{array}{l}\text { a brazo: } \\
\text { a braccia }\end{array}$ & $\begin{array}{c}\text { a braccio: } \\
\text { improvisando }\end{array}$ & $\begin{array}{l}\text { a brazo: } \\
\text { a braccia }\end{array}$ & $\begin{array}{l}\text { a braccio: } \\
\text { improvisando }\end{array}$ & $\begin{array}{c}\text { a brazo: } \\
-\end{array}$ & $\begin{array}{c}\text { a braccio: } \\
-\end{array}$ \\
\hline $\begin{array}{l}\text { abrir boca: } \\
\text { stuzzicare } \\
\text { l'appetito }\end{array}$ & $\begin{array}{c}\text { non aprire } \\
\text { bocca: } \\
\text { no abrir la } \\
\text { boca, no decir } \\
\text { esta boca es } \\
\text { mía, no soltar } \\
\text { prenda }\end{array}$ & $\begin{array}{c}\text { abrir (o hacer) } \\
\text { boca: } \\
\text { stuzzicare } \\
\text { l'appetito }\end{array}$ & $\begin{array}{c}\text { non aprire } \\
\text { bocca: } \\
\text { no decir esta } \\
\text { boca es mía, } \\
\text { no abrir la } \\
\text { boca }\end{array}$ & $\begin{array}{c}\text { no abrir la } \\
\text { boca: } \\
\text { non aprire } \\
\text { bocca, restare } \\
\text { in silenzio }\end{array}$ & $\begin{array}{l}\text { non aprire } \\
\text { bocca: } \\
\text { o rechistar, no } \\
\text { abrir la boca, } \\
\text { no decir nada }\end{array}$ \\
\hline $\begin{array}{l}\text { a manos lle- } \\
\text { nas: a piene } \\
\text { mani, in ab- } \\
\text { bondanza }\end{array}$ & $\begin{array}{l}\text { *a mani piene: } \\
\text { a manos llenas }\end{array}$ & $\begin{array}{c}\text { *a manos } \\
\text { llenas: } \\
\text { a piene mani }\end{array}$ & $\begin{array}{c}\text { a mani piene: } \\
\text { a manos lle- } \\
\text { nas }\end{array}$ & $\begin{array}{c}\text { a manos lle- } \\
\text { nas: } \\
-\end{array}$ & $\begin{array}{l}\text { a mani piene: } \\
\text { a manos llenas }\end{array}$ \\
\hline $\begin{array}{c}\text { *en sus barbas: } \\
\text { in barba a, sotto } \\
\text { il naso }\end{array}$ & $\begin{array}{c}\text { in barba a: } \\
\text { a despecho de, } \\
\text { en su barba/cara }\end{array}$ & $\begin{array}{c}\text { en las barbas de } \\
\text { alguien: } \\
\text { in faccia a } \\
\text { qualcuno }\end{array}$ & $\begin{array}{c}\text { in barba a: } \\
\text { a despecho de }\end{array}$ & $\begin{array}{l}\text { *en las barbas } \\
\text { de alguien: } \\
\text { in barba a qual- } \\
\text { cuno }\end{array}$ & $\begin{array}{c}\text { *in barba a } \\
\text { qualcuno: en las } \\
\text { barbas de al- } \\
\text { guien }\end{array}$ \\
\hline $\begin{array}{l}\text { mano a mano: } \\
\text { in collaborazio- } \\
\text { ne, in competi- } \\
\text { zione }\end{array}$ & $\begin{array}{l}\text { a mano a mano: } \\
\text { a medida que, } \\
\text { poco a poco }\end{array}$ & $\begin{array}{c}\text { mano a mano: } \\
-\end{array}$ & $\begin{array}{c}\text { a mano a mano: } \\
-\end{array}$ & $\begin{array}{l}\text { mano a mano: } \\
\text { (a solas dos per- } \\
\text { sonas), a tu per } \\
\text { tu; (colaboran- }\end{array}$ & $\begin{array}{l}\text { a mano a mano: } \\
\text { a medida que }\end{array}$ \\
\hline
\end{tabular}




\begin{tabular}{|c|c|c|c|c|c|}
\hline \multicolumn{2}{|c|}{ TAM } & \multicolumn{2}{|c|}{ GA } & \multicolumn{2}{|c|}{ VOX } \\
\hline ESP-IT & IT-ES & PESP-IT & IT-ESP & ESP-IT & IT-ESP \\
\hline & & & & $\begin{array}{l}\text { do) in collabo- } \\
\text { razione; (com- } \\
\text { pitiendo) in } \\
\text { competizione }\end{array}$ & \\
\hline $\begin{array}{c}\text { meter } \\
\text { mano:mettere } \\
\text { le mani su, } \\
\text { metterci mano, } \\
\text { palpare }\end{array}$ & $\begin{array}{l}\text { mettere mano: } \\
\text { echar mano a }\end{array}$ & $\begin{array}{l}\text { meter mano: } \\
\text { (a algo) mette- } \\
\text { re mano a } \\
\text { qlco; (a al- } \\
\text { guien) palpeg- } \\
\text { giare qlcu. }\end{array}$ & $\begin{array}{c}\text { mettere mano: } \\
\text { meter(le) } \\
\text { mano a algo }\end{array}$ & $\begin{array}{c}\text { meter mano: } \\
\text { intervenire }\end{array}$ & $\begin{array}{c}\text { mettere mano: } \\
-\end{array}$ \\
\hline $\begin{array}{c}\text { ir cabeza aba- } \\
\text { jo: } \\
-\end{array}$ & $\begin{array}{l}\text { * a testa bas- } \\
\text { sa: cabizbajo }\end{array}$ & $\begin{array}{c}\text { *cabeza aba- } \\
\text { jo: prono, a } \\
\text { testa in giù }\end{array}$ & $\begin{array}{l}\text { * a testa bas- } \\
\text { sa: cabizbajo }\end{array}$ & $\begin{array}{c}\text { *cabeza aba- } \\
\text { jo: a testa in } \\
\text { giù }\end{array}$ & $\begin{array}{c}\text { a testa bassa: } \\
-\end{array}$ \\
\hline
\end{tabular}

A partir de esta tabla se deduce que:

- El Tam es el diccionario más regular en la incorporación de las UF. Se puede afirmar que es el más avanzado desde un punto de vista cuantitativo; no obstante, desde un punto de vista cualitativo son patentes sus incongruencias. En muchas ocasiones no se toman en consideración las discrepancias de significado entre ambas lenguas: es el caso de en sus barbas, expresión que se traduce de forma literal, creando así confusión respecto al verdadero significado con el que se utiliza en cada uno de los dos idiomas.

- El Vox es el más desprovisto de UF. A ello hay que añadir su gran imprecisión al proporcionar equivalencias. Así, omite la locución abrir boca, aunque sí incluye el modismo no abrir boca, con un significado que en este caso resulta idéntico al del italiano.

- El GA se sitúa en un punto intermedio entre el Tam y el Vox, tanto por cantidad de UF registradas como por la selección de sus equivalencias. Es el único diccionario que aporta indicaciones más precisas a propósito de las equivalencias aparentes meter mano y mettere mano.

Ninguno de los tres diccionarios se puede considerar satisfactorio en cuanto a la calidad de las equivalencias presentadas. Un caso concreto de inexactitud que afecta a los tres viene representado por la locución darse con la cabeza contra la pared. En español existen dos expresiones afines que corresponden a dos significados distintos: por un lado, dar con la cabeza en las paredes significa 'precipitarse en un negocio con perjuicio para la propia persona', por otro, darse con la cabeza en la pared o en las paredes indica 'desesperarse por algo que ha ido mal'. Sin embargo, esta diferencia no se especifica en ninguno. Además, en la sección de italiano del Vox y en correspondencia con el ejemplo non sapere dove sbattersi la testa, se recoge no tener dónde volver la cabeza, lo que generaría una nueva equivalencia, que entre otros aspectos, resultaría 
poco apropiada, puesto que la expresión española quiere decir 'no hallar auxilio, carecer de todo favor'.

\section{CONCLUSIÓN}

En este análisis, nuestro propósito ha sido el de observar los diferentes tipos de traducción de la fraseología en el marco de la lexicografía bilingüe hispano-italiana. Como resultado, se nos revela de manera incuestionable que la frecuente falta de correspondencias exactas entre las dos lenguas ocasiona ciertos errores e incongruencias que son motivo de desorientación para los usuarios. A este propósito, Quiroga (2006: 105) manifiesta cierta perplejidad: «la falta de información pragmática en el tratamiento del significado de las UF, en los diccionarios italiano-español estudiados ${ }^{1}$, constituye una laguna que deberá llenar la lexicografía del futuro».

Somos conscientes de la dificultad que entraña la traducción de las unidades fraseológicas a otra lengua, pero reconocemos también la importancia que poseen estas unidades en el proceso de aprendizaje de una L2. Por consiguiente, aunque la tarea sea delicada, su buena consecución se basa en un principio claro: en los repertorios bilingües es necesario elegir la estrategia de traducción más acertada, porque, como recuerda Navarro (2005: 441), «el objetivo fundamental de los diccionarios bilingües es ofrecer un equivalente en L2 que llegue a cubrir todas las propiedades semánticas, pragmáticas y comunicativas de la UF de L1».

Por lo general, los criterios de traducción no son uniformes y es difícil averiguar a partir de qué principios se ha actuado. No se halla tampoco en los diccionarios ninguna indicación acerca de la eficacia pragmática de las unidades registradas.

Podríamos finalmente considerar como mayor desacierto la imposibilidad de saber cuál es el equivalente de traducción de uso más frecuente. Lamentablemente, en ninguno de estos diccionarios aparece una guía con este tipo de aclaraciones y el usuario tiene que conformarse con una información limitada, sin poder contar con detalles adicionales.

De todo ello se deduce que no es fácil manejar y obtener beneficio del diccionario bilingüe por lo que respecta a la fraseología. Sin embargo, su utilización puede resultar constructiva, ya que fomenta la reflexión, evitando que los usuarios recurran a este instrumento de manera superficial y mecánica. Finalizamos en este punto nuestro análisis, con la firma determinación de investigar y explorar nuevos aspectos alrededor de este tema en sucesivos estudios.

\section{BIBLIOGRAFÍA}

Carneado Moré, Z. (1985): Estudios de fraseología. Cuba: Academia de Ciencias de Cuba, Instituto de Literatura y Lingüística.

CASAdei, F. (2001): Breve Dizionario di Linguistica. Roma: Carocci.

CoRpas Pastor, G. (1996): Manual de fraseología española. Madrid: Gredos.

${ }^{1}$ Los diccionarios analizados por Quiroga son repertorios bilingües del siglo XX, entre los cuales destacan el Ambruzzi, el Carbonell y el Tam. 
García Yebra, V. (1983): En torno a la traducción. Madrid: Gredos.

ILINÁ, N. (2000): La fraseología española contemporánea: estado de la cuestión. Facultad de Lenguas Extranjeras adjunta a la Universidad Estatal Lomonosov de Moscù. http://hispanismo.cervantes.es/documentos/lina.pdf

LiVERANI, E. (2008): "Il Grande dizionario di spagnolo-italiano, italiano-spagnolo (2004) de L. Tam”. En F. San Vicente (Ed.), Textos fundamentales de la lexicografía italoespañola (19172007). Monza: Polimetrica Publisher, pp. 439-494.

NAVARro, C. (2005): "La fraseología en los diccionarios bilingües español/italiano". En Actas del XXIII Congreso AISPI (Palermo, 6-8 ottobre, 2005), vol.2: Lingüística contrastiva entre el italiano y las lenguas ibéricas / Linguistica contrastiva tra italiano e lingue iberiche, a cura di L. Blini, M. V. Calvi e A. Cancellier. Madrid: Instituto Cervantes, pp. 428-445.

- (2008): Aspectos de fraseología contrastiva Español - Italiano. Verona: Edizioni Fiorini.

OlZA Moreno, I. (2006): "Las partes del cuerpo humano como bases metonímicas en la fraseología metalingüística del español", en Actes del VII Congrés de Lingüística General. Barcelona: Publicacions i Edicions de la Universitat de Barcelona, edición en CD-ROM, 1-18.

QUiroga, P. (2006): Fraseología ítalo-española. Aspectos de lingüística aplicada y contrastiva. Granada: Método.

ToROP, P. (1995): La traduzione totale. Tartu: Edizioni dell'Università di Tartu.

\section{DICCIONARIOS}

AA. VV. (2009): I Grandi Dizionari Spagnolo, (Spagnolo •Italiano, Italiano • Spagnolo). Levis (TN): Garzanti Linguistica.

Real Academia Española (2001): Diccionario de la Lengua Española, 22. ${ }^{a}$ ed., Madrid: Espasa Calpe.

SAÑÉ, S., y SchePISI, G. (2005): VoX - Il dizionario Spagnolo-Italiano/El diccionario ItalianoEspañol, Bolonia: Zanichelli.

TAM, L. (1997): Dizionario spagnolo-italiano-Diccionario italiano-español. Milán: Hoepli.

Zingarelli, N. (2011): Vocabolario della lingua italiana. Bolonia: Zanichelli. 